\title{
BMJ Open Association of napping and night-time sleep with impaired glucose regulation, insulin resistance and glycated haemoglobin in Chinese middle-aged adults with no diabetes: a cross- sectional study
}

\author{
Huang Baoying, ${ }^{1}$ Chen Hongjie, ${ }^{2}$ Qiu Changsheng, ${ }^{3}$ Wu Peijian, ${ }^{1}$ Lin Qingfei, ${ }^{3}$ \\ Lin Yinghua, ${ }^{1}$ Huang Huibin, ${ }^{2}$ Liang Jixing, ${ }^{2}$ Li Liantao, ${ }^{2}$ Chen Ling, ${ }^{2}$ Tang Kaka, ${ }^{2}$ \\ Chen Zichun, ${ }^{1}$ Lin Lixiang, ${ }^{2}$ Lu Jieli, ${ }^{4}$ Bi Yufang, ${ }^{4}$ Ning Guang, ${ }^{4}$ Zhu Penli, ${ }^{2}$ \\ Wen Junping, ${ }^{2}$ Chen Gang $^{2}$
}

To cite: Baoying $\mathrm{H}$, Hongjie C, Changsheng $Q$, et al. Association of napping and night-time sleep with impaired glucose regulation, insulin resistance and glycated haemoglobin in Chinese middle-aged adults with no diabetes: a crosssectional study. BMJ Open 2014:4:e004419.

doi:10.1136/bmjopen-2013004419

- Prepublication history for this paper is available online. To view these files please visit the journal online (http://dx.doi.org/10.1136/ bmjopen-2013-004419).

$\mathrm{HB}, \mathrm{CH}, \mathrm{QC}, \mathrm{WP}$ and $\mathrm{LQ}$ contributed equally to the study.

Received 14 November 2013 Revised 1 July 2014

Accepted 3 July 2014 CrossMark

For numbered affiliations see end of article.

Correspondence to Dr Gang Chen; chengang18@yahoo.com

\section{ABSTRACT}

Objective: To assess associations between napping and night-time sleep duration with impaired glucose regulation, insulin resistance (IR) and glycated haemoglobin (HbA1c).

Design: Cross-sectional study.

Setting: Fujian Province, China, from June 2011 to January 2012.

Participants: This study enrolled 9028 participants aged 40-65 years. Data of 7568 participants with no diabetes were included for analysis. Type 2 diabetes was defined applying WHO criteria.

Outcome measures: Participants' daytime napping and night-time sleep duration data were collected using a standardised self-reported Chinese-language questionnaire about sleep frequency and quality. Anthropometric and laboratory parameters were also measured. IR was defined as a HOMA-IR index value $>2.50$. ORs and $95 \% \mathrm{Cls}$ were derived from multivariate logistic regression models.

Results: Participants (mean age $51.1 \pm 7.0$ years) included 3060 males and 4508 females with average night-time sleep of $7.9 \mathrm{~h}$. A higher proportion of males napped than females. After adjustment for potential confounders, ORs for $\mathrm{HbA} 1 \mathrm{c}>6.0 \%$ were 1.28 and 1.26 for those napping $\leq 1 \mathrm{~h}$ and $>1 \mathrm{~h}(\mathrm{p}=0.002$ and $p=0.018$ ), respectively. Statistically significant differences in IR between nappers and non-nappers were only marginal clinically. Odds for $\mathrm{HbA} 1 \mathrm{c}>6.0 \%$ were significantly lower in participants with longer night-time sleep durations than in the reference group ( $>8 \mathrm{~h}$ vs $6-8 \mathrm{~h}$ ). Odds for IR were significantly lower in participants whose night-time sleep hours deviated from the reference group ( $<6 \mathrm{~h},>8 \mathrm{~h}$ vs $6-8 \mathrm{~h}$ )

Conclusions: Chinese middle-aged adults with no diabetes who napped had higher HbA1C and IR; those with shorter night-time sleep durations had increased HbA1c. Night-time sleep hours that are either $<6$ or $>8$

\section{Strengths and limitations of this study}

This study showed that middle-aged Chinese adults with out diabetes who reported napping and shorter night-time sleep had higher HbA1C. Daytime napping was also associated with higher odds of insulin resistance. Night-time sleep hours that deviate from the reference 6 to $8 \mathrm{~h}$ show association with lower odds for insulin resistance.

- The strength of the results is the simultaneous assessment of the pathophysiological pathways of hyperglycaemia and insulin resistance together with glycaemic level.

- The major strengths of the current study include the large sample size and the wide range of demographic, socioeconomic and health-related data collected in the analysis to account for potential confounders.

- Limitations: because of its cross-sectional design, the direction of causality could not be determined from our analysis. Second, because self-reported questionnaires were used to obtain data on night-time sleep duration and napping, the study lacks an objective sleep measurement.

tend to be associated with lower odds for IR. Further studies are necessary to determine the underlying clinical significance and mechanisms behind these associations.

\section{INTRODUCTION}

Sleep loss is a common condition in today's society. Sleep duration per night is reported to have declined by $1.5-2 \mathrm{~h}$ in different 
populations over the last few decades, but data from the same study do not support that all adults need more sleep. ${ }^{1}$ Additionally, several experimental and epidemiological studies have shown that shortened or altered sleep may adversely affect glucose homoeostasis, including decreased glucose tolerance and increased insulin resistance (IR). ${ }^{2-4}$

Daytime napping is also common in many parts of the world, particularly in China, where napping is a wellaccepted behaviour in all age groups as part of a regular routine and healthy lifestyle. ${ }^{5}{ }^{6}$ However, little is understood about the effects of napping on health, especially on glucose metabolism, including IR, impaired glucose regulation (IGR) and glycated haemoglobin (HbAlc).

Diabetes remains a critical public health challenge, especially with the related increased risk of cardiovascular-related mortality. The prevalence of diabetes in China has increased remarkably from $2.5 \%$ in 1994 to $9.7 \%$ in 2010, making China one of the countries with the largest diabetes burden worldwide. ${ }^{7}$ IGR, IR and $\mathrm{HbAlc}$ are associated with increased risk of cardiovascular diseases and diabetes..$^{8-10}$

Therefore, the purpose of the present cross-sectional analysis was to assess the associations between daytime napping and night-time sleep duration with IGR, IR and HbA1c in middle-aged Chinese adults with no diabetes.

\section{METHODS}

\section{Study population}

We conducted this cross-sectional study from June 2011 to January 2012 in Fujian Province, China. Study participants were selected from the cohort of the REACTION study (Risk evaluation of cancers in Chinese diabetic individuals: a longitudinal study), which investigated the association between diabetes and cancer in 259657 Chinese adults aged 40 years and older in 25 communities across mainland China from 2011 to $2012 .{ }^{11}$ For the present study, a total of 9028 participants aged 40-65 years completed a standardised questionnaire and blood samples were collected. The data of 7568 participants who met the inclusion criteria (ie, absence of diabetes) were retained for further analysis. The remaining 1460 patients with a diagnosis of diabetes or incomplete data were excluded, including 1247 patients with diabetes, 31 patients with missing data of napping and night-time sleep and 182 patients without laboratory and anthropometric data. All investigators received special training (eg, acquiring a basic understanding of the questionnaire and being trained to take measurements for blood pressure, body height, weight and waist circumference) before the investigation. The study received approval from the Endocrinology Branch of the Chinese Medical Association, and all participants gave written informed consent.

\section{Data collection}

Data, including demographics, medical history and lifestyle factors from all 7568 participants were recorded. Height and weight, waist and hip circumference and blood pressure for each participant were determined by a single physician. Waist circumference was measured at the level of the umbilicus and hip circumference at the widest part of the buttocks according to standardised WHO criteria and the recommendations of expert consultation. ${ }^{12}$ Altogether four physicians conducted all examinations, each participating for 2 months at a time over the data collection period. Body mass index (BMI) was calculated as weight $(\mathrm{kg})$ divided by the square of height $(\mathrm{m})$. The waist-to-hip ratio (WHR) was calculated as the ratio of waist-to-hip circumference. Blood pressure was measured three times using an automatic sphygmomanometer after the participants had been resting for $5 \mathrm{~min}$ in a sitting position before each measurement; the mean of the three readings was used for analysis. After at least an 8-h overnight fast, venous blood samples were drawn at 0 and $2 \mathrm{~h}$ during a $75 \mathrm{~g}$ oral glucose tolerance test. Blood glucose, including fasting blood glucose (FBG) and $2 \mathrm{~h}$ plasma glucose $(\mathrm{PG})$, was measured using the glucose oxidase method. Fasting serum insulin (FINS), triglycerides (TG), total cholesterol (TC), high-density lipoprotein cholesterol (HDL-C) and low-density lipoprotein cholesterol (LDL-C) levels were determined using chemiluminescence methods. HbA1c was measured by highperformance liquid chromatography.

Participants were asked to self-report the average hours and minutes of daytime napping and night-time sleep per day. Napping habits were categorised as no napping, $\leq 1 \mathrm{~h}$ and $>1 \mathrm{~h}$ napping per day. Night-time sleep duration was divided into three categories: $<6 \mathrm{~h}$, $6-8 \mathrm{~h}$ and $\geq 8 \mathrm{~h}$. Participants' snoring habits were also recorded during the interview.

\section{Definitions and diagnostic criteria}

Type 2 diabetes was defined according to WHO criteria $^{13}$ as fasting plasma glucose $\geq 7.0 \mathrm{mmol} / \mathrm{L}$ and/or $2 \mathrm{~h}$ postprandial glucose $\geq 11.1 \mathrm{mmol} / \mathrm{L}$, or as having been diagnosed with type 2 diabetes. IGR was defined as $6.1 \leq$ fasting plasma glucose $<7 \mathrm{mmol} / \mathrm{L}$ and/or $7.8 \leq 2 \mathrm{~h}$ postprandial glucose $<11.1 \mathrm{mmol} / \mathrm{L}$. HOMA-IR was calculated as fasting insulin $(\mathrm{mU} / \mathrm{L}) *$ fasting plasma glucose $(\mathrm{mmol} / \mathrm{L}) / 22.5,{ }^{14}$ and IR was defined as HOMA-IR values $>2.50 .{ }^{15} \mathrm{HbAlc}$ was the preferred test for monitoring glucose control, and HbAlc >6.0\%, based on WHO guidelines for $\mathrm{HbAlc}^{13}$ and as previously described in the literature, ${ }^{10}$ was applied as an independent predictor of subsequent cardiovascular disease in middle-aged adults with no diabetes.

A diagnosis of hypertension was based on the Seventh Joint National Commission recommendation of systolic blood pressure $\geq 140 \mathrm{~mm} \mathrm{Hg}$ and/or diastolic blood pressure $\geq 90 \mathrm{~mm} \mathrm{Hg}$ or having been diagnosed with hypertension or use of antihypertension treatment. Dyslipidaemia was defined as having one or more of the following: $\mathrm{TC}>6.2 \mathrm{mmol} / \mathrm{L}, \mathrm{TG}>2.3 \mathrm{mmol} / \mathrm{L}$, LDL-C $>4.1 \mathrm{mmol} / \mathrm{L}$, HDL-C $<0.91 \mathrm{mmol} / \mathrm{L}$ or $\mathrm{TC} / \mathrm{HDL}-\mathrm{C}$ $>5 .{ }^{16}$ Obesity was defined as BMI $\geq 30 \mathrm{~kg} / \mathrm{m}^{2}$ and overweight was defined as $25 \leq \mathrm{BMI}<30 \mathrm{~kg} / \mathrm{m}^{2}$. 


\section{Statistical analysis}

Clinical data were presented as mean \pm SD for normally distributed data, as median with IQR for skew distributed data and as number with percentage for categorical data. Since only $0.1 \%$ of participants reported $\geq 2 \mathrm{~h}$ of napping, we combined participants reporting $>1 \mathrm{~h}$ and $<1 \mathrm{~h}$ napping for analysis, and participants reporting no nap were used as the reference group. One-way analysis of variance, $\chi^{2}$ test or non-parametric test were used to compare differences between categories of napping (eg, no nap, $\leq 1 \mathrm{~h}$ and $>1 \mathrm{~h}$ ) or night-time sleep (eg, $<6 \mathrm{~h}$, $6-8 \mathrm{~h},>8 \mathrm{~h}$ ). The Bonferroni correction was applied for the comparisons of pair-wise groups. Binary logistic regression analysis was constructed to assess the ORs of HbA1c $<6.0 \%$, IGR and IR associated with napping and night-time sleep. The first regression model was unadjusted. The second models included napping time and night-time sleep (adjusting for one another). The third models were further adjusted for age and gender. To prevent the effect of latent confounders, another 11 variables were selected by the forward conditional method before entering into the fourth model (FBG, hypertension, family history of diabetes (FHD), dyslipidaemia, smoking status, alcohol status, frequency of snoring, level of physical activity, education levels, BMI and WHR). All data analyses were conducted with SPSS V.17.0 statistical software package (SPSS, Chicago, Illinois, USA). All $\mathrm{p}$ values $<0.05$ (two-sided) indicated statistical significance.

\section{RESULTS}

Of the 7568 participants (mean age: $51.1 \pm 7.0$ years), 3060 males and 4508 females were included in the analysis; the percentage of females was greater than that of males in all three groups and males were only marginally older in the napping group compared to the other two groups. Participants' average night-time sleep was $7.9 \mathrm{~h} ; 46.5 \%$ did not nap while $36.6 \%$ and $16.9 \%$ napped $\leq 1 \mathrm{~h}$ and $>1 \mathrm{~h}$, respectively. Table 1 shows baseline characteristics by napping categories. When compared with the no napping group or less napping $(>1 \mathrm{~h})$ group, those who napped over $1 \mathrm{~h}$ were more likely to have lower FBG, higher $2 \mathrm{~h}$ PG, lower HDL-C, higher WHR and higher snoring frequency; they also were more likely to be smokers and alcohol users, although only minor differences were found. Compared with the no napping group, the two groups who napped 1 and $>1 \mathrm{~h}$ were found to have significant differences, including lower SBP, higher FINS, higher TG, higher HbAlc and higher HOMA-IR, but these statistically significant differences were still marginal clinically. In addition, fewer participants with night-time sleep over $8 \mathrm{~h}$ were observed in the two groups that reported 1 and $>1 \mathrm{~h}$ of napping compared to the no napping group $(27.1 \%$ and $27.9 \%$ vs $45.5 \%$; table 1 ).

Demographic and clinical characteristics were also assessed by group based on night-time sleep duration (table 2). Participants who reported sleeping $<6 \mathrm{~h}$ per night were more likely to nap than those reporting $\geq 8 \mathrm{~h}$ of night-time sleep, who were less likely to nap. Significant differences were found in SBP, FBG, FINS, HDL-C, WHR and HbA1c between those with night-time sleep durations of more than $8 \mathrm{~h}$ compared to those with night-time sleep durations of less than $6 \mathrm{~h}$ or from 6 to $8 \mathrm{~h}$, however, some differences may not be considered clinically meaningful in terms of participants' health and/or sleep status (table 2).

As shown in table 3, participants who reported napping had a higher risk of HbAlc $>6.0 \%$, IGR and IR. Compared with the no napping group, the crude OR of HbAlc $>6.0 \%$ for those with napping time $>1 \mathrm{~h}$ was 1.24 $(\mathrm{p}=0.018)$. After adjusting for other variables (eg, nighttime sleep, age, gender, FBG, hypertension, dyslipidaemia, smoking status, alcohol status, education levels, BMI and WHR), the adjusted ORs of HbAlc $>6.0 \%$ for those with napping times $\leq 1 \mathrm{~h}$ and $>1 \mathrm{~h}$ were 1.28 $(\mathrm{p}=0.002)$ and $1.26(\mathrm{p}=0.018)$, respectively. Similar associations were observed between IR and napping, the adjusted ORs of IR for those with napping times $\leq 1 \mathrm{~h}$ and $>1 \mathrm{~h}$ were $1.55 \quad(\mathrm{p}<0.001)$ and $1.69 \quad(\mathrm{p}<0.001)$, respectively. No associations were observed between IGR and napping (table 3 ).

In terms of night-time sleep, participants with longer night-time sleep durations $(>8 \mathrm{~h})$ were less likely to have HbAlc $>6.0 \%$ and IR compared to those with $6-8 \mathrm{~h}$ night-time sleep (table 4). Compared with the reference group (individuals with 6-8 h sleep per night), the crude OR for HbAlc $>6.0 \%$ was $1.47(p<0.001)$ among participants with night-time sleep $<6 \mathrm{~h}$. In contrast, the crude OR was $0.70(\mathrm{p}<0.001)$ for participants with night-time sleep of $\geq 8 \mathrm{~h}$. The association between longer night-time sleep duration and HbAlc $>6.0 \%$ remained significant after adjusting for other variables $(\mathrm{OR}=0.69$ and 0.57 with $\mathrm{p}<0.001)$. Compared with the reference group, the crude ORs for IGR did not reach statistical significance, but the adjusted ORs showed that participants with shorter nighttime sleep $(<6 \mathrm{~h})$ were less likely to have IGR, where $\mathrm{OR}=0.77 \quad(\mathrm{p}=0.042)$ when adjusted for age, gender and napping, and $\mathrm{OR}=0.74(\mathrm{p}=0.020)$ when further adjusted for other variables selected by the forward conditional method (hypertension, FDH, dyslipidaemia, smoking status, alcohol status, snoring, education level, BMI and WHR). The crude OR showed that participants with $<6 \mathrm{~h}$ night sleep were less likely to have IR $(\mathrm{OR}=0.69$, $\mathrm{p}=0.048)$. The ORs of such associations remained statistically significant after adjustments for daytime napping, age and gender, but the OR $(0.70, \mathrm{p}=0.096)$ did not remain statistically significant after further adjustments for other variables selected by the forward conditional method (ie, FBG, hypertension, dyslipidaemia, alcohol status, BMI and WHR). However, a significant association between longer night-time sleep $(>8 \mathrm{~h})$ and IR was observed in the final model; the adjusted OR of 0.84 ( $p=0.049$ ) showed that participants with night-time sleep $>8 \mathrm{~h}$ were less likely to have IR (table 4 ). 
Table 1 Characteristics of the study participants according to daytime napping categories

\begin{tabular}{|c|c|c|c|c|}
\hline & \multicolumn{3}{|l|}{ Napping } & \multirow[b]{2}{*}{ p Value } \\
\hline & $\begin{array}{l}\text { No napping, } \\
n=3517(46.5 \%)\end{array}$ & $\begin{array}{l}1 \text { h napping, } \\
n=2769(36.6 \%)\end{array}$ & $\begin{array}{l}\text { >1 h napping, } \\
\mathrm{n}=1282(16.9 \%)\end{array}$ & \\
\hline \multicolumn{5}{|l|}{ Gender* } \\
\hline Male & 1353 (38.5\%) & $1117(40.3 \%)$ & $590(46.0 \%) \dagger, \ddagger$ & \multirow[t]{2}{*}{$<0.001$} \\
\hline Female & $2164(61.5 \%)$ & $1652(59.7 \%)$ & $692(54.0 \%)$ & \\
\hline Age (year)§ & $50.88(6.98)$ & $51.04(6.96)$ & 51.63 (7.03)†,‡ & 0.004 \\
\hline SBP $(\mathrm{mm} \mathrm{Hg}) \S$ & $131.92(18.42)$ & $130.04(18.25) \dagger$ & $130.10(19.35) \dagger$ & $<0.001$ \\
\hline $\mathrm{DBP}(\mathrm{mm} \mathrm{Hg}) \S$ & 77.17 (10.87) & $77.41(10.78)$ & 76.92 (11.40) & 0.382 \\
\hline $\mathrm{FBG}(\mathrm{mmol} / \mathrm{L}) \S$ & $5.40(0.59)$ & $5.28(0.62) \dagger$ & $5.21(0.65) \dagger$ & $<0.001$ \\
\hline 2 h PG $(\mathrm{mmol} / \mathrm{L}) \S$ & $6.38(1.65)$ & $6.58(1.65) \dagger$ & $6.74(1.67) \dagger, \ddagger$ & $<0.001$ \\
\hline FINS (uIU/mL)ף & $5.40(3.70,7.80)$ & $6.00(4.30,8.40) \dagger$ & $6.20(4.30,8.70) \dagger$ & $<0.001$ \\
\hline HDL-C (mmol/L)§ & $1.42(0.35)$ & $1.37(0.34) \dagger$ & $1.33(0.35) \dagger, \ddagger$ & $<0.001$ \\
\hline LDL-C (mmol/L)§ & $2.92(0.83)$ & $2.94(0.82)$ & $2.95(0.85)$ & 0.365 \\
\hline $\mathrm{TC}(\mathrm{mmol} / \mathrm{L}) \S$ & $5.04(1.06)$ & $5.06(1.07)$ & $5.05(1.13)$ & 0.681 \\
\hline $\mathrm{TG}(\mathrm{mmol} / \mathrm{L}) \S$ & $1.48(1.12)$ & 1.59 (1.14)† & $1.62(1.14) \dagger$ & $<0.001$ \\
\hline $\mathrm{WHR}^{2}$ & $0.864(0.059)$ & $0.866(0.061)$ & $0.873(0.077) \dagger, \ddagger$ & $<0.001$ \\
\hline BMI $\left(\mathrm{kg} / \mathrm{m}^{2}\right) \S$ & $23.86(3.14)$ & $23.88(3.01)$ & 24.07 (3.18) & 0.116 \\
\hline $\mathrm{HbA1c}(\%) \S$ & $5.61(0.43)$ & $5.65(0.42) \dagger$ & $5.67(0.43) \dagger$ & $<0.001$ \\
\hline HOMA-IRף & $1.29(0.87,1.89)$ & $1.40(0.98,1.98) \dagger$ & $1.40(0.96,2.02) \dagger$ & $<0.001$ \\
\hline \multicolumn{5}{|l|}{ Dyslipidaemia* } \\
\hline Normal & 2643 (75.2\%) & 1950 (70.4\%) & 879 (68.6\%) & \multirow[t]{2}{*}{$<0.001$} \\
\hline Abnormal & $873(24.8 \%)$ & $818(29.6 \%) \dagger$ & $402(31.4 \%) \dagger$ & \\
\hline \multicolumn{5}{|l|}{ Smokers ${ }^{\star}$} \\
\hline No & 2671 (75.9\%) & 2178 (78.7\%) & $922(71.9 \%)$ & \multirow[t]{2}{*}{$<0.001$} \\
\hline Yes & $846(24.1 \%)$ & $591(21.3 \%)$ & $360(28.1 \%) \dagger, \ddagger$ & \\
\hline \multicolumn{5}{|l|}{ Alcohol users* } \\
\hline No & 2886 (82.1\%) & $2234(80.7 \%)$ & $989(77.1 \%)$ & \multirow[t]{2}{*}{0.002} \\
\hline Yes & 631 (17.9\%) & $535(19.3 \%)$ & 293 (22.9\%)†,‡ & \\
\hline \multicolumn{5}{|l|}{$\mathrm{FHD}^{*}$} \\
\hline No & 3311 (94.1\%) & $2540(91.7 \%)$ & 1195 (93.2\%) & \multirow[t]{2}{*}{0.002} \\
\hline Yes & $206(5.9 \%)$ & $229(8.3 \%) \dagger$ & $87(6.8 \%)$ & \\
\hline \multicolumn{5}{|l|}{ Physical activity ${ }^{\star}$} \\
\hline No & 3327 (94.6\%) & $2353(85.0 \%)$ & $1068(83.3 \%)$ & \multirow[t]{2}{*}{$<0.001$} \\
\hline Yes & $190(5.4 \%)$ & $416(15.0 \%) \dagger$ & $214(16.7 \%) \dagger$ & \\
\hline \multicolumn{5}{|l|}{ Snoring* } \\
\hline Often & $676(19.2 \%)$ & $521(18.8 \%)$ & $297(23.2 \%) \dagger, \ddagger$ & \multirow[t]{3}{*}{$<0.001$} \\
\hline Seldom & $1043(29.7 \%)$ & $1020(36.8 \%)$ & 442 (34.5\%) & \\
\hline Never & $1798(51.1 \%)$ & $1228(44.3 \%)$ & $543(42.4 \%)$ & \\
\hline \multicolumn{5}{|l|}{ Education level* } \\
\hline Low & 1635 (46.5\%) & $882(31.9 \%)$ & 508 (39.6\%) & \multirow[t]{3}{*}{$<0.001$} \\
\hline Medium & 1656 (47.1\%) & 1366 (49.3\%) & 639 (49.8\%) & \\
\hline High & $226(6.4 \%)$ & $521(18.8 \%) \dagger$ & 135 (10.5\%)†, & \\
\hline \multicolumn{5}{|l|}{ Hypertension* } \\
\hline No & $2310(65.7 \%)$ & $1837(66.3 \%)$ & $865(67.5 \%)$ & \multirow[t]{2}{*}{0.508} \\
\hline Yes & 1207 (34.3\%) & $932(33.7 \%)$ & 417 (32.5\%) & \\
\hline Obesity* & & & & \\
\hline Normal & $2341(66.7 \%)$ & $1831(66.2 \%)$ & 827 (64.7\%) & 0.032 \\
\hline Overweight & 1025 (29.2\%) & $854(30.9 \%)$ & 394 (30.8\%) & \\
\hline Obesity & $143(4.1 \%)$ & $81(2.9 \%) \dagger$ & $58(4.5 \%) \ddagger$ & \\
\hline Night-time Sleep* & & & & \\
\hline$<6$ & 127 (3.6\%) & $143(5.2 \%)$ & 76 (5.9\%) & $<0.001$ \\
\hline $6-8$ & 1790 (50.9\%) & $1875(67.7 \%)$ & $848(66.1 \%)$ & \\
\hline$>8$ & $1600(45.5 \%)$ & 751 (27.1\%)†,‡ & $358(27.9 \%) \dagger$ & \\
\hline $\begin{array}{l}\text { Obesity was definec } \\
\text { *Data are presentec } \\
\text { †Indicates a signific } \\
\text { łIndicates a signific } \\
\text { §Data are presente } \\
\text { IData are presente } \\
\text { BMI, body mass ind } \\
\text { HbA1c, glycated ha } \\
\text { resistance; LDL-C, } \\
\text { triglycerides; WHR, }\end{array}$ & $\begin{array}{l}30 \mathrm{~kg} / \mathrm{m}^{2} \text {. } \\
\text { and percentage. } \\
\text { nce compared to the } \mathrm{g} \\
\text { nce compared to the } \mathrm{g} \\
\text { I and SD. } \\
\text { an and IQR due to skev } \\
\text { diastolic blood pressure } \\
\text {; HDL-C, high-density } \\
\text { y lipoprotein cholestero } \\
\text { ip ratio. }\end{array}$ & $\begin{array}{l}\text { lapping. } \\
\text { ping } 1 \mathrm{~h} \text {. } \\
\text { n. } \\
\text { ing blood glucose; FHD } \\
\text { cholesterol; HOMA-IR, th } \\
\text { na glucose; SBP, systoli }\end{array}$ & $\begin{array}{l}\text { tory of diabetes; FINS, } \\
\text { stasis model assessme } \\
\text { essure; TC, total choles }\end{array}$ & \\
\hline
\end{tabular}


Table 2 Characteristics of study participants according to night-time sleep categories

\begin{tabular}{|c|c|c|c|c|}
\hline & \multicolumn{3}{|l|}{ Night-time Sleep } & \multirow[b]{2}{*}{ p Value } \\
\hline & $<6 \mathrm{~h}, \mathrm{n}=346(4.6 \%)$ & $6-8 \mathrm{~h}, \mathrm{n}=4513(59.6 \%)$ & $>8 \mathrm{~h}, \mathrm{n}=2709(35.8 \%)$ & \\
\hline \multicolumn{5}{|l|}{ Gender* } \\
\hline Male & $150(43.4 \%)$ & $1756(38.9 \%)$ & $1154(42.6 \%) \dagger$ & 0.005 \\
\hline Female & $196(56.6 \%)$ & $2757(61.1 \%)$ & $1555(57.4 \%)$ & \\
\hline Age (year) $\ddagger$ & $53.23(6.46)$ & $50.90(6.94) \S$ & $51.06(7.08) \S$ & $<0.001$ \\
\hline $\mathrm{SBP}(\mathrm{mm} \mathrm{Hg}) \ddagger$ & $129.88(17.81)$ & $129.68(18.27)$ & $133.13(18.88) \dagger, \S$ & $<0.001$ \\
\hline $\mathrm{DBP}(\mathrm{mm} \mathrm{Hg}) \ddagger$ & 76.66 (10.98) & 76.89 (11.05) & $77.84(10.71) \dagger$ & 0.001 \\
\hline FBG $(\mathrm{mmol} / \mathrm{L}) \ddagger$ & $5.20(0.63)$ & $5.28(0.63) \S$ & $5.41(0.59) \dagger, \S$ & $<0.001$ \\
\hline $2 \mathrm{~h} \mathrm{PG}(\mathrm{mmol} / \mathrm{L}) \ddagger$ & $6.50(1.59)$ & $6.54(1.67)$ & $6.46(1.66)$ & 0.134 \\
\hline FINS (uIU/mL)ף & $5.90(4.40,7.80)$ & $5.90(4.20,8.40)$ & $5.40(3.70,7.80) \dagger, \S$ & $<0.001$ \\
\hline HDL-C (mmol/L) $\ddagger$ & $1.36(0.35)$ & $1.37(0.34)$ & $1.41(0.35) \dagger, \S$ & $<0.001$ \\
\hline LDL-C (mmol/L) $\ddagger$ & $2.96(0.83)$ & $2.94(0.82)$ & $2.92(0.85)$ & 0.474 \\
\hline $\mathrm{TC}(\mathrm{mmol} / \mathrm{L}) \ddagger$ & $5.11(1.10)$ & $5.04(1.07)$ & $5.05(1.09)$ & 0.516 \\
\hline $\mathrm{TG}(\mathrm{mmol} / \mathrm{L}) \ddagger$ & $1.54(1.18)$ & $1.55(1.14)$ & $1.54(1.10)$ & 0.971 \\
\hline WHR & $0.879(0.082)$ & $0.864(0.063) \S$ & $0.869(0.062) \dagger, \S$ & $<0.001$ \\
\hline BMI $\left(\mathrm{kg} / \mathrm{m}^{2}\right) \ddagger$ & $24.18(3.27)$ & $23.93(3.11)$ & $23.81(3.05)$ & 0.059 \\
\hline $\mathrm{HbA} 1 \mathrm{c}(\%) \ddagger$ & $5.73(0.43)$ & $5.65(0.42) \S$ & $5.60(0.42) \dagger, \S$ & $<0.001$ \\
\hline HOMA-IRף & $1.35(1.00,1.84)$ & $1.38(0.95,1.99)$ & $1.30(0.87,1.90) \dagger$ & $<0.001$ \\
\hline \multicolumn{5}{|l|}{ Dyslipidaemia* } \\
\hline Normal & $249(72.0 \%)$ & 3250 (72.1\%) & 1973 (72.8\%) & 0.771 \\
\hline Abnormal & 97 (28.0\%) & 1260 (27.9\%) & 736 (27.2\%) & \\
\hline \multicolumn{5}{|l|}{ Smokers $^{*}$} \\
\hline No & 247 (71.4\%) & 3521 (78.0\%) & 2003 (73.9\%) & $<0.001$ \\
\hline Yes & $99(28.6 \%)$ & $992(22.0 \%) \S$ & $706(26.1 \%) \dagger$ & \\
\hline \multicolumn{5}{|l|}{ Alcohol users* } \\
\hline No & 275 (79.5\%) & 3644 (80.7\%) & 2190 (80.8\%) & 0.819 \\
\hline Yes & $71(20.5 \%)$ & 869 (19.3\%) & $519(19.2 \%)$ & \\
\hline \multicolumn{5}{|l|}{$\mathrm{FHD}^{*}$} \\
\hline No & 318 (91.9\%) & 4167 (92.3\%) & 2561 (94.5\%) & 0.001 \\
\hline Yes & $28(8.1 \%)$ & $346(7.7 \%)$ & $148(5.5 \%) \dagger$ & \\
\hline \multicolumn{5}{|l|}{ Physical activity* } \\
\hline No & 294 (85.0\%) & 3920 (86.9\%) & $2534(93.5 \%)$ & $<0.001$ \\
\hline Yes & $52(15.0 \%)$ & $593(13.1 \%)$ & $175(6.5 \%) \dagger, \S$ & \\
\hline \multicolumn{5}{|l|}{ Snoring* } \\
\hline Often & 77 (22.3\%) & 900 (19.9\%) & $517(19.1 \%)$ & 0.003 \\
\hline Seldom & $110(31.8 \%)$ & $1560(34.6 \%)$ & $835(30.8 \%)$ & \\
\hline Never & 159 (46.0\%) & 2053 (45.5\%) & $1357(50.1 \%) \dagger$ & \\
\hline \multicolumn{5}{|l|}{ Education level* } \\
\hline Low & $154(44.5 \%)$ & 1541 (34.1\%) & 1330 (49.1\%) & $<0.001$ \\
\hline Medium & $164(47.4 \%)$ & 2307 (51.1\%) & 1190 (43.9\%) & \\
\hline High & $28(8.1 \%)$ & $665(14.7 \%) \S$ & $189(7.0 \%) \dagger$ & \\
\hline \multicolumn{5}{|l|}{ Hypertension* } \\
\hline No & 220 (63.6\%) & 3085 (68.4\%) & 1707 (63.0\%) & $<0.001$ \\
\hline Yes & $126(36.4 \%)$ & $1428(31.6 \%)$ & $1002(37.0 \%) \dagger$ & \\
\hline \multicolumn{5}{|l|}{ Obesity* } \\
\hline Normal & $226(65.3 \%)$ & 2968 (65.8\%) & 1805 (66.9\%) & 0.840 \\
\hline Overweight & $105(30.3 \%)$ & 1372 (30.4\%) & 796 (29.5\%) & \\
\hline Obesity & $15(4.3 \%)$ & $170(3.8 \%)$ & $97(3.6 \%)$ & \\
\hline \multicolumn{5}{|l|}{ Napping* } \\
\hline No napping & 127 (36.7\%) & 1790 (39.7\%) & $1600(59.1 \%)$ & $<0.001$ \\
\hline $1 \mathrm{~h}$ & $143(41.3 \%)$ & 1875 (41.5\%) & $751(27.7 \%) \dagger, \S$ & \\
\hline$>1 \mathrm{~h}$ & $76(22.0 \%)$ & $848(18.8 \%)$ & $358(13.2 \%) \dagger, \S$ & \\
\hline
\end{tabular}

Obesity was defined as $\mathrm{BMI} \geq 30 \mathrm{~kg} / \mathrm{m}^{2}$.

*Data are presented by count and percentage.

†Indicates a significant difference compared to the group of night-time sleep 6-8 $\mathrm{h}$.

fData are presented by mean and SD.

§Indicates a significant difference compared to the group of night-time sleep $<6 \mathrm{~h}$.

IData are presented by median and IQR due to skewed distribution.

BMI, body mass index; DBP, diastolic blood pressure; FBG, fasting blood glucose; FHD, family history of diabetes; FINS, fasting insulin;

$\mathrm{HbA1c}$, glycated haemoglobin; HDL-C, high-density lipoprotein cholesterol; HOMA-IR, the homoeostasis model assessment of insulin

resistance; LDL-C, low-density lipoprotein cholesterol; PG, plasma glucose; SBP, systolic blood pressure; TC, total cholesterol; TG,

triglycerides; WHR, Waist-to-hip ratio. 
Table 3 The crude and adjusted ORs of $\mathrm{HbA} 1 \mathrm{c}>6.0 \%$, impaired glucose regulation and insulin resistance according to daytime napping

\begin{tabular}{|c|c|c|c|c|c|c|c|c|c|}
\hline $\begin{array}{l}\text { Dependent } \\
\text { variable }\end{array}$ & $\begin{array}{l}\text { Independent } \\
\text { variable }\end{array}$ & $\begin{array}{l}\text { Crude OR } \\
(95 \% \mathrm{Cl})\end{array}$ & p Value & $\begin{array}{l}\text { Adjusted OR } \\
(95 \% \mathrm{Cl})\end{array}$ & p Value & $\begin{array}{l}\text { Adjusted OR† } \\
(95 \% \mathrm{Cl})\end{array}$ & p Value & $\begin{array}{l}\text { Adjusted OR } \\
(95 \% \mathrm{Cl})\end{array}$ & p Value \\
\hline \multirow[t]{4}{*}{$\mathrm{HbA} 1 \mathrm{C}>6.0 \%$} & Napping & & & & & & & & \\
\hline & No nap & 1.0 & & 1.0 & & 1.0 & & 1.0 & \\
\hline & $\leq 1 \mathrm{~h}$ & $1.13(0.98$ to 1.30$)$ & 0.102 & 1.05 (0.91 to 1.22$)$ & 0.491 & 1.05 (0.91 to 1.22$)$ & 0.516 & 1.28 (1.09 to 1.49$)$ & $0.002 \S$ \\
\hline & $>1 \mathrm{~h}$ & 1.24 (1.04 to 1.48$)$ & $0.018 \S$ & $1.16(0.97$ to 1.38$)$ & 0.112 & 1.13 (0.94 to 1.36$)$ & 0.186 & 1.26 (1.04 to 1.53$)$ & $0.018 \S$ \\
\hline \multirow[t]{4}{*}{ IGR } & Napping & & & & & & & & \\
\hline & No nap & 1.0 & & 1.0 & & 1.0 & & 1.0 & \\
\hline & $\leq 1 \mathrm{~h}$ & 1.05 (0.94 to 1.17$)$ & 0.394 & 1.06 (0.95 to 1.19$)$ & 0.313 & 1.06 (0.94 to 1.18$)$ & 0.345 & $1.08(0.96$ to 1.21$)$ & 0.214 \\
\hline & $>1 \mathrm{~h}$ & $1.13(0.98$ to 1.30$)$ & 0.085 & 1.14 (0.99 to 1.32$)$ & 0.064 & 1.12 (0.97 to 1.29$)$ & 0.123 & $1.11(0.95$ to 1.28$)$ & 0.181 \\
\hline \multirow[t]{4}{*}{ Insulin resistance } & Napping & & & & & & & & \\
\hline & No nap & 1.0 & & 1.0 & & 1.0 & & 1.0 & \\
\hline & $\leq 1 \mathrm{~h}$ & 1.22 (1.05 to 1.42$)$ & $0.012 \S$ & 1.21 (1.04 to 1.41$)$ & $0.016 \S$ & $1.22(1.05$ to 1.43$)$ & $0.011 \S$ & 1.55 (1.30 to 1.85$)$ & $<0.001 \S$ \\
\hline & $>1 \mathrm{~h}$ & $1.33(1.10$ to 1.61$)$ & $0.003 \S$ & 1.33 (1.10 to 1.60$)$ & $0.004 \S$ & 1.37 (1.13 to 1.66$)$ & $0.001 \S$ & 1.69 (1.35 to 2.12$)$ & $<0.001 \S$ \\
\hline \multicolumn{10}{|c|}{$\begin{array}{l}\text { HbA1c, glycated haemoglobin; IGR, impaired glucose regulation. } \\
\text { *Adjusted by night-time sleep. } \\
\text { †Further adjusted by age and gender. }\end{array}$} \\
\hline
\end{tabular}




\begin{tabular}{|c|c|c|c|c|c|c|c|c|c|}
\hline Dependent variable & Independent variable & $\begin{array}{l}\text { Crude } \\
\text { OR }(95 \% \mathrm{Cl})\end{array}$ & p Value & $\begin{array}{l}\text { Adjusted } \\
\mathrm{OR}^{\star}(95 \% \mathrm{Cl})\end{array}$ & p Value & $\begin{array}{l}\text { Adjusted } \\
\text { ORt }(95 \% \mathrm{Cl})\end{array}$ & p Value & $\begin{array}{l}\text { Adjusted } \\
\text { OR }(95 \% \mathrm{Cl})\end{array}$ & p Value \\
\hline \multirow[t]{4}{*}{$\mathrm{HbA} 1 \mathrm{C}>6.0 \%$} & Night-time sleep & & & & & & & & \\
\hline & $<6 \mathrm{~h}$ & 1.47 (1.12 to 1.92$)$ & $0.005 \S$ & $1.46(1.12$ to 1.91$)$ & $0.006 \S$ & 1.27 (0.97 to 1.67 ) & 0.088 & 1.25 (0.94 to 1.66$)$ & 0.122 \\
\hline & $6-8 \mathrm{~h}$ & 1.0 & & 1.0 & & 1.0 & & 1.0 & \\
\hline & $>8 \mathrm{~h}$ & 0.70 (0.60 to 0.80$)$ & $<0.001 \S$ & 0.71 (0.61 to 0.82 ) & $<0.001 \S$ & 0.69 (0.60 to 0.80$)$ & $<0.001 \S$ & 0.57 (0.49 to 0.66$)$ & $<0.001 \S$ \\
\hline \multirow[t]{4}{*}{ IGR } & Night-time sleep & & & & & & & & \\
\hline & $<6 \mathrm{~h}$ & 0.85 (0.66 to 1.10$)$ & 0.214 & 0.85 (0.66 to 1.09$)$ & 0.202 & 0.77 (0.60 to 0.99$)$ & $0.042 \S$ & 0.74 (0.57 to 0.95$)$ & $0.020 \S$ \\
\hline & $6-8 \mathrm{~h}$ & 1.0 & & 1.0 & & 1.0 & & 1.0 & \\
\hline & $>8 \mathrm{~h}$ & $1.02(0.92$ to 1.14$)$ & 0.656 & $1.04(0.94$ to 1.16$)$ & 0.471 & $1.04(0.93$ to 1.15$)$ & 0.526 & $1.00(0.89$ to 1.11$)$ & 0.940 \\
\hline \multirow[t]{4}{*}{ Insulin resistance } & Night-time sleep & & & & & & & & \\
\hline & $<6 \mathrm{~h}$ & 0.69 (0.47 to 1.00$)$ & $0.048 \S$ & $0.68(0.47$ to 0.99$)$ & $0.043 \S$ & 0.68 (0.47 to 0.99$)$ & $0.044 \S$ & 0.70 (0.46 to 1.06$)$ & 0.096 \\
\hline & $6-8 \mathrm{~h}$ & 1.0 & & 1.0 & & 1.0 & & 1.0 & \\
\hline & $>8 \mathrm{~h}$ & 0.90 (0.78 to 1.04$)$ & 0.147 & 0.94 (0.81 to 1.09$)$ & 0.384 & $0.95(0.82$ to 1.11$)$ & 0.518 & $0.84(0.71$ to 1.00$)$ & $0.049 \S$ \\
\hline \multicolumn{10}{|c|}{$\begin{array}{l}\text { HbA1c, glycated haemoglobin; IGR, impaired glucose regulation. } \\
\text { *Adjusted by daytime napping. } \\
\text { †Further adjusted by age and gender. } \\
\text { fThe } 11 \text { variables were stepwise entered into the final model by forward conditional method (FBG, hypertension, FHD, dyslipidaemia, smoking status, alcohol status, frequency of snoring, } \\
\text { physical activity, education levels, BMI and WHR); for the final model for HbA1C, the following eight variables were included, FBG, hypertension, dyslipidaemia, smoking status, alcohol status, } \\
\text { education level, BMI and WHR; for the final model for IGR, the following nine variables were included, hypertension, FDH, dyslipidaemia, smoking status, alcohol status, snoring, education level } \\
\text { BMl and WHR, where FBG was excluded since it is part of the definition of IGR; for the final model for IR, the following six variables were included, FBG, hypertension, dyslipidaemia, alcohol } \\
\text { status, BMl and WHR. } \\
\text { \$p<0.05 indicated a significant association with the dependent variable. }\end{array}$} \\
\hline
\end{tabular}




\section{DISCUSSION}

Results of the present cross-sectional study of Chinese middle-aged adults without diabetes revealed that participants with shorter night-time sleep $(<6 \mathrm{~h})$ were more likely to have HbA1c $>6.0 \%$ and IGR compared with these values in persons reporting $6-8 \mathrm{~h}$ of night-time sleep after adjusting for potential confounding factors. More importantly, we found that daytime napping $(\leq 1$ or $>1 \mathrm{~h}$ ) was significantly adversely associated with $\mathrm{HbA} 1 \mathrm{c}>6.0 \%$ and IR.

To the best of our knowledge, the present study is the first extensive investigation of daytime napping and night-time sleep in conjunction with glucose metabolism (ie, HbAlc, IGR and IR) in middle-aged adults with out diabetes. In previous epidemiologic studies regarding associations of napping with different health parameters, exceptionally few studies assessed associations with diabetes or prediabetes. A cross-sectional study, The Guangzhou Biobank Cohort Study, suggested that participants reporting frequent naps (4-6 days/week or daily) were $37 \%$ or $15 \%$ more likely to have impaired fasting glucose and $36 \%$ or $28 \%$ more likely to have diabetes after adjusting for various potential confounders. ${ }^{17}$ However, unlike our study, that previous study assessed the effect of napping in terms of napping frequency and did not evaluate other markers of glucose metabolism, including HbAlc and IR. ${ }^{17}$ In the present study, we observed altered glucose metabolism in middle-aged adult participants without diabetes who reported napping. In western countries, napping appears to be less common and may compensate for night-time sleep loss or be induced by adverse health status such as in persons with diabetes. However, in China, daytime napping is a very traditional practice common in all ages. In fact, previous studies have suggested that nighttime sleep duration was not significantly associated with napping duration. ${ }^{17} 18$ Considering this, we excluded participants with diabetes defined by self-reports and biochemical test results. We considered that associations between daytime napping and results of HbAlc, IGR and IR were not likely due to reverse causation.

Several mechanisms other than obesity may help to explain the associations between napping and these markers of glucose metabolism. Waking from daytime napping increases sympathetic nervous system activity, which could activate the renin-angiotensin system and increase cortisol levels, thereby modulating IR and associated hyperglycaemia. ${ }^{19} 20$ However, the mechanism involved in these relationships may not be fully explained. Moreover, individuals, particularly those reporting $>1 \mathrm{~h}$ daily napping, are likely to have unique lifestyle factors (eg, type of work, working hours, personalities, etc) beyond napping that may influence glucose metabolism. Further prospective or experimental studies are needed, such as studies including more categories of duration (eg, 15, 30, 60, $90 \mathrm{~min}$, etc), to better explore the possible underlying mechanisms of these associations.
Results of the present analysis also found that night-time sleep duration was significantly associated with HbA1c and IGR. The lack of associations between IR and night-time sleep duration among our participants without diabetes is in agreement with a prior report in participants with out diabetes in China. ${ }^{21}$ In another previous study, even though the participants were involved in more rigid glucose control, as in persons with diabetes, the association between napping and IR remained insignificant. ${ }^{22}$ In contrast, a recent cohort study in rural Chinese participants without diabetes also yielded significant adverse associations between self-reported short night-time sleep and IR after adjusting for adiposity and other potential confounders, but only in women, not in men. ${ }^{23}$ Discrepancies between those results and other previous results may be attributed to different sample sizes, different grouping of night-time sleep durations or different definitions of IR by HOMA-IR. However, the previous cross-sectional study observed the same result as ours by conducting multivariable linear regression with night-time sleep and HOMA-IR as continuous variables. ${ }^{22}$ At the same time, it should be noted that although statistically significant differences were shown in certain measured parameters between nappers and non-nappers, many were not clinically significant differences. For example, the difference between a mean age of $50.9 \pm 7$ years in non-nappers vs $51 \pm 7$ years in the $<1 \mathrm{~h}$ napping group is obviously not clinically meaningful; it indicates only a slight difference in age. Similarly, the differences in SBP were 131.9 in non-nappers vs 130.0 and 130.1 in nappers $(<1 \mathrm{~h}$ and $>1 \mathrm{~h})$, which are statistically significant but were still only slightly different, not indicating a clinically significant difference. Again, although significant differences were found in FBG, FINS, HDL-C, WHR and HbAlc between groups, the actual differences would not be considered clinically meaningful in terms of participants' health and/or sleep status. Some differences, such as the HOMA-IR results between longest and shortest sleepers, were clearly significant. However, this may be a result of the way participants were grouped, that is, showing trends toward possible diabetes risk in Chinese adults who nap. Although the analysis in the present study had sufficient power due to the large sample size, the lack of clinically significant differences in conjunction with statistically significant differences between groups can only be explained as a result of confounding variables in the models and not to actual intrinsic differences between the groups. Further longitudinal study is definitely needed, including comparisons between the sleeping and napping habits of participants with and without diabetes.

Consistent with a recent study, ${ }^{24}$ and especially important among results of the present study, participants with short night-time sleep duration were more likely to have IGR and higher HbAlc. However, in contrast to the present study, a Japanese study reported a U-shaped association between night-time sleep and HbAlc $>6.5 \% .^{25}$ Several underlying biological mechanisms may explain the association between night-time sleep and disordered glucose metabolism. For example, an experimental study suggested that night-time 
sleep deprivation led to decreased levels of the appetitesuppressing hormone, leptin, increased levels of the appetite-stimulating hormone, ghrelin, sympathetic hyperactivity and elevated evening cortisol levels through activation of the hypothalamic-pituitary-adrenal axis, ${ }^{2}$ which may inhibit pancreatic function leading to reduced glucose tolerance and IR. ${ }^{26}$

The major strengths of the current study include the large sample size and the wide range of demographic, socioeconomic and health-related data that were collected in the analysis to account for potential confounders. However, this study also has limitations. First, because of its cross-sectional design, the direction of causality could not be determined from our analysis. Second, the absence of dietary data may be described as a study limitation given the associations between diet, sleep and hyperglycaemia, which is mediated through appetite-associated hormones. $^{2}{ }^{25}$ Third, as self-reported questionnaires were used to obtain data on night-time sleep duration and napping, the study lacks objective sleep measurement using polysomnography or actigraphy, which were not feasible given our large sample and misclassifications may possibly occur. Nevertheless, an earlier study comparing subjective and actigraphic measurement of sleep found good correlations between self-reported sleep duration and objective measurements. ${ }^{27}$ Finally, data were not obtained on the quality of night-time sleep, including the possible presence of insomnia or obstructive sleep apnoea (OSA), which previous studies have suggested were associated with impaired glucose metabolism ${ }^{28} 29$ and napping. ${ }^{6}$ Based on a previous study defining OSA on the basis of daytime sleepiness and disordered breathing during sleep, which includes snoring, ${ }^{30}$ we accounted for the frequency of self-reported or spouse-reported snoring as a marker of OSA risk, and the inclusion of snoring in the regression model did not change the associations. Nevertheless, future studies must include polysomnography data to support related findings.

In summary, results of the present cross-sectional study indicate that Chinese middle-aged adult participants without diabetes reporting daytime napping had significantly elevated HbA1c, IGR and IR, and those with shorter night-time sleep durations had higher HbAlc and impaired glucose regulation. Further experimental studies are warranted to identify the mechanism underlying these associations.

\section{Author affiliations \\ ${ }^{1}$ Department of Endocrinology, Ningde Municipal Hospital, Fujian Medical University, Ningde, China \\ ${ }^{2}$ Department of Endocrinology, Fujian Provincial Hospital Key Laboratory of Endocrinology, Fujian Medical University, Fuzhou, China \\ ${ }^{3}$ Department of Osteology, Wuyishan Municipal Hospital, Fujian Provincial Hospital, Wuyishan, China \\ ${ }^{4}$ Department of Endocrinology, Ruijin Hospital, Shanghai Jiaotong University School of Medicine, Shanghai, China}

Contributors HB was involved in study concept, study design, manuscript review and is the guarantor. $\mathrm{CH}$ was involved in literature research, data acquisition, data analysis, statistical analysis, manuscript preparation and manuscript editing. QC was involved in definition of intellectual content, literature research, data acquisition, data analysis, manuscript editing and manuscript review. WP was involved in literature research, data acquisition, data analysis, manuscript editing and manuscript review. LQ, LY, HH and LJ were involved in literature research, data acquisition, data analysis. LL, CL, TK and CZ were involved in data acquisition and data analysis. LL was involved in literature research and manuscript review. LJ and BY were involved in study design and definition of intellectual content. NG was involved in study concept, study design and is the guarantor. ZP and WJ were involved in study concept, definition of intellectual content, manuscript review and are guarantors. CG was involved in study concept, definition of intellectual content, manuscript editing, manuscript review and is the guarantor.

Funding This study was supported by grants from the Chinese Medical Association Foundation and Chinese Endocrine Society (12020240314), National Natural Science Foundation of China (81270874), Natural Science Foundation of Fujian Province (2011J06012), Provincial Health and Family Planning Commission of Fujian Province (2013-ZQN-ZD-3). The funders had no role in study design, data collection and analysis, decision to publish, or preparation of the manuscript.

\section{Competing interests None.}

\section{Patient consent Obtained.}

Ethics approval This study was approved by the institutional review board of Fujian Provincial Hospital.

Provenance and peer review Not commissioned; externally peer reviewed.

Data sharing statement No additional data are available.

Open Access This is an Open Access article distributed in accordance with the Creative Commons Attribution Non Commercial (CC BY-NC 3.0) license, which permits others to distribute, remix, adapt, build upon this work noncommercially, and license their derivative works on different terms, provided the original work is properly cited and the use is non-commercial. See: http:// creativecommons.org/licenses/by-nc/3.0/

\section{REFERENCES}

1. Jean-Louis G, Kripke DF, Ancoli-Israel S. Sleep and quality of well-being. Sleep 2002;23:1115-21.

2. Spiegel K, Leproult R, Van Cauter E. Impact of sleep debt on metabolic and endocrine function. Lancet 1999;354:1435-9.

3. Xu Q, Song $Y$, Hollenbeck $A$, et al. Day napping and short night sleeping are associated with higher risk of diabetes in older adults. Diabetes Care 2010;33:78-83.

4. Rafalson L, Donahue RP, Stranges S, et al. Short sleep duration is associated with the development of impaired fasting glucose: the Western New York Health Study. Ann Epidemiol 2010;20:883-9.

5. Lan TY, Lan TH, Wen CP, et al. Nighttime sleep, Chinese afternoon nap, and mortality in the elderly. Sleep 2007;30:1105-10.

6. Liu X, Liu L. Sleep habits and insomnia in a sample of elderly persons in China. Sleep 2005;28:1579-87.

7. Yang SH, Dou KF, Song WJ. Prevalence of diabetes among men and women in China. N Engl J Med 2010;362:2425-6; author reply 2426.

8. Expert Committee on the Diagnosis and Classification of Diabetes Mellitus. Report of the expert committee on the diagnosis and classification of diabetes mellitus. Diabetes Care 1997;20:1183-97.

9. Coutinho M, Gerstein HC, Wang Y, et al. The relationship between glucose and incident cardiovascular events. A metaregression analysis of published data from 20 studies of 95,783 individuals followed for 12.4 years. Diabetes Care 1999;22:233-40.

10. Selvin E, Steffes MW, Zhu H, et al. Glycated hemoglobin, diabetes, and cardiovascular risk in nondiabetic adults. N Engl J Med 2010;362:800-11.

11. Bi Y, Lu J, Wang W, et al. Cohort profile: risk evaluation of cancers in Chinese diabetic individuals: a longitudinal (REACTION) study. J Diabetes 2014;6:147-57.

12. World Health Organization. Waist circumference and waist-hip ratio: report of a WHO expert Consultation. Geneva: World Health Organization, 2008.

13. World Health Organization. Definition, diagnosis and classification of diabetes mellitus and its complications. Use of glycated haemoglobin $(\mathrm{HbA1c})$ in the diagnosis of diabetes mellitus. WHO/ NMH/CHP/CPM/11.1. Geneva: World Health Organization, 2011.

14. Matthews DR, Hosker JP, Rudenski AS, et al. Homeostasis model assessment: insulin resistance and beta-cell function from fasting plasma glucose and insulin concentrations in man. Diabetologia 1985;28:412-19. 
15. Wang T, Li M, Chen B, et al. Urinary bisphenol A (BPA) concentration associates with obesity and insulin resistance. J Clin Endocrinol Metab 2012;97:E223-7.

16. Lee ZS, Chan JC, Yeung VT, et al. Plasma insulin, growth hormone, cortisol, and central obesity among young Chinese type 2 diabetic patients. Diabetes Care 1999;22:1450-7.

17. Lam KB, Jiang CQ, Thomas GN, et al. Napping is associated with increased risk of type 2 diabetes: the Guangzhou Biobank Cohort Study. Sleep 2010;33:402-7.

18. Goldman SE, Hall M, Boudreau R, et al. Association between nighttime sleep and napping in older adults. Sleep 2008;31:733-40.

19. Smolensky MH, Hermida RC, Castriotta RJ, et al. Role of sleep-wake cycle on blood pressure circadian rhythms and hypertension. Sleep Med 2007;8:668-80.

20. Rasch B, Dodt C, Molle M, et al. Sleep-stage-specific regulation of plasma catecholamine concentration. Psychoneuroendocrinology 2007:32:884-91.

21. Zuo H, Shi Z, Yuan B, et al. Interaction between physical activity and sleep duration in relation to insulin resistance among non-diabetic Chinese adults. BMC Public Health 2012:12:247.

22. Knutson KL, Van Cauter E, Zee P, et al. Cross-sectional associations between measures of sleep and markers of glucose metabolism among subjects with and without diabetes: the Coronary Artery Risk Development in Young Adults (CARDIA) Sleep Study. Diabetes Care 2011;34:1171-6.
23. Liu R, Zee PC, Chervin RD, et al. Short sleep duration is associated with insulin resistance independent of adiposity in Chinese adult twins. Sleep Med 2011;12:914-19.

24. Byberg S, Hansen AL, Christensen DL, et al. Sleep duration and sleep quality are associated differently with alterations of glucose homeostasis. Diabet Med 2012;29:e354-60.

25. Nakajima H, Kaneita Y, Yokoyama E, et al. Association between sleep duration and hemoglobin A1c level. Sleep Med 2008;9:745-52.

26. Reaven GM, Lithell H, Landsberg L. Hypertension and associated metabolic abnormalities - the role of insulin resistance and the sympathoadrenal system. N Engl J Med 1996;334:374-81.

27. Lockley SW, Skene DJ, Arendt J. Comparison between subjective and actigraphic measurement of sleep and sleep rhythms. J Sleep Res 1999;8:175-83.

28. Vgontzas AN, Liao D, Pejovic S, et al. Insomnia with objective short sleep duration is associated with type 2 diabetes: a population-based study. Diabetes Care 2009;32:1980-5.

29. Priou P, Le Vaillant M, Meslier N, et al. IRSR Sleep Cohort Group. Independent association between obstructive sleep apnea severity and glycated hemoglobin in adults without diabetes. Diabetes Care 2012;35:1902-6.

30. Jennum P, Riha RL. Epidemiology of sleep apnoea/hypopnoea syndrome and sleep-disordered breathing. Eur Respir J 2009;33:907-14. 